\title{
3-Methylglutaconic Acidemia in Smith-Lemli-Opitz Syndrome
}

\author{
RICHARD I. KELLEY and LISA KRATZ \\ Division of Neurogenetics, Kennedy Krieger Institute and Department of Pediatrics, \\ Johns Hopkins University School of Medicine, Baltimore, Maryland 21205
} \begin{abstract}
metabolism, we have speculated that some patients with 3-methylglutaconic aciduria may have defects of polyisoprenoid or sterol biosynthesis leading to overflow of isoprenoid precursors to 3-methylglutaconate via the mevalonate shunt. We therefore measured plasma levels of 3-methylglutaconic acid in patients with a known defect of sterol biosynthesis, Smith-Lemli-Opitz syndrome, and found that the patients with the lowest cholesterol
\end{abstract}

levels had abnormally increased plasma levels of 3-methylglutaconic acid, similar in magnitude to those of patients with idiopathic 3-methylglutaconic aciduria. This finding suggests that some patients with unexplained 3-methylglutaconic aciduria may have defects of isoprenoid or sterol biosynthesis underlying their abnormal organic aciduria. (Pediatr Res 37: 671-674, 1995)

\author{
Abbreviations \\ 3MGC, 3-methylglutaconic acid \\ SLOS, Smith-Lemli-Opitz syndrome \\ 7DHC, 7-Dehydrocholesterol \\ HMG, 3-hydroxy-3-methylglutarate
}

Although $3 \mathrm{MGC}$ is best known as a CoA intermediate in the catabolism of L-leucine, the urinary excretion of 3MGC appears to be independent of the metabolism of leucine in the majority of patients with abnormal $3 \mathrm{MGC}$-aciduria $(1,2)$. Except in several patients with mild neurologic abnormalities caused by a genetic deficiency of 3-methylglutaconyl-CoA hydratase $(3,4)$, provocative testing including fasting, oral loading with L-leucine, and high protein diets have had little effect on the excretion of 3MGC, even in patients with 50- to 100 -fold increased excretion of $3 \mathrm{MGC}$. And whereas a few patients with excessive $3 \mathrm{MGC}$ aciduria have been found to have a primary defect of mitochondrial energy metabolism $(5,6)$, most searches for a specific metabolic lesion in patients with $3 \mathrm{MGC}$ aciduria prove fruitless.

Because patients with the syndrome of X-linked cardiomyopathy and neutropenia (Barth syndrome) have both increased 3 MGC aciduria and hypocholesterolemia (1), we have speculated that some idiopathic syndromes associated with $3 \mathrm{MGC}$ aciduria may be caused by defects of sterol or isoprenoid

Received for rapid publication October 27, 1994; accepted January 31, 1995.

Correspondence and reprint requests: Richard I. Kelley. M.D., Ph.D., Kennedy Krieger Institute, 707 North Broadway, Baltimore, MD 21205.

Supported, in part, by grants HD-07107, HD-10981, and DK-44933 from the National Institutes of Health.

This work was presented, in part, at the 1994 Annual Meeting of the American Pediatric Society and the Society for Pediatric Research, Seattle, WA, May 2-5. metabolism, causing overflow of mevalonate carbon through the mevalonate shunt pathway to 3MGC (Fig. 1). In the mevalonate shunt first proposed in 1974 by Edmond and Popjak (7), dimethylallyl pyrophosphate is dephosphorylated to the free alcohol, oxidized in two steps to 3-methylcrotonic acid, and then activated with CoA to form 3-methylcrotonyl$\mathrm{CoA}$, the precursor of $3 \mathrm{MGC}-\mathrm{CoA}$ in the normal leucine catabolic pathway. Other plausible links between the two pathways at the level of higher order isoprenoids, such as geraniol and farnesol, have been described by Schroepfer (8). Also possible is formation of $3 \mathrm{MGC}-\mathrm{CoA}$ by dehydration of HMG-CoA, either spontaneously or by reverse activity of 3MGC-CoA hydratase. Physiologic evidence that the mevalonate shunt or a related shunt pathway is significant in humans and other mammals has been provided by several laboratories (9-12), but whether the $3 \mathrm{MGC}$ in some patients with abnormal $3 \mathrm{MGC}$-aciduria derives from isoprenoid precursors is unknown.

The well-known congenital malformation syndrome, SLOS (13), has recently been found to have a block in cholesterol biosynthesis at the level of $3 \beta$-hydroxysteroid- $\Delta^{7}$-reductase, the terminal enzyme of the Kandutsch-Russell (14) pathway for synthesis of cholesterol (15). The unusually high plasma levels of 7DHC found in patients with SLOS suggested to us that if $3 \mathrm{MGC}$ were a product of a mevalonate shunt pathway, then the high levels of cholesterol precursors likely to occur in 

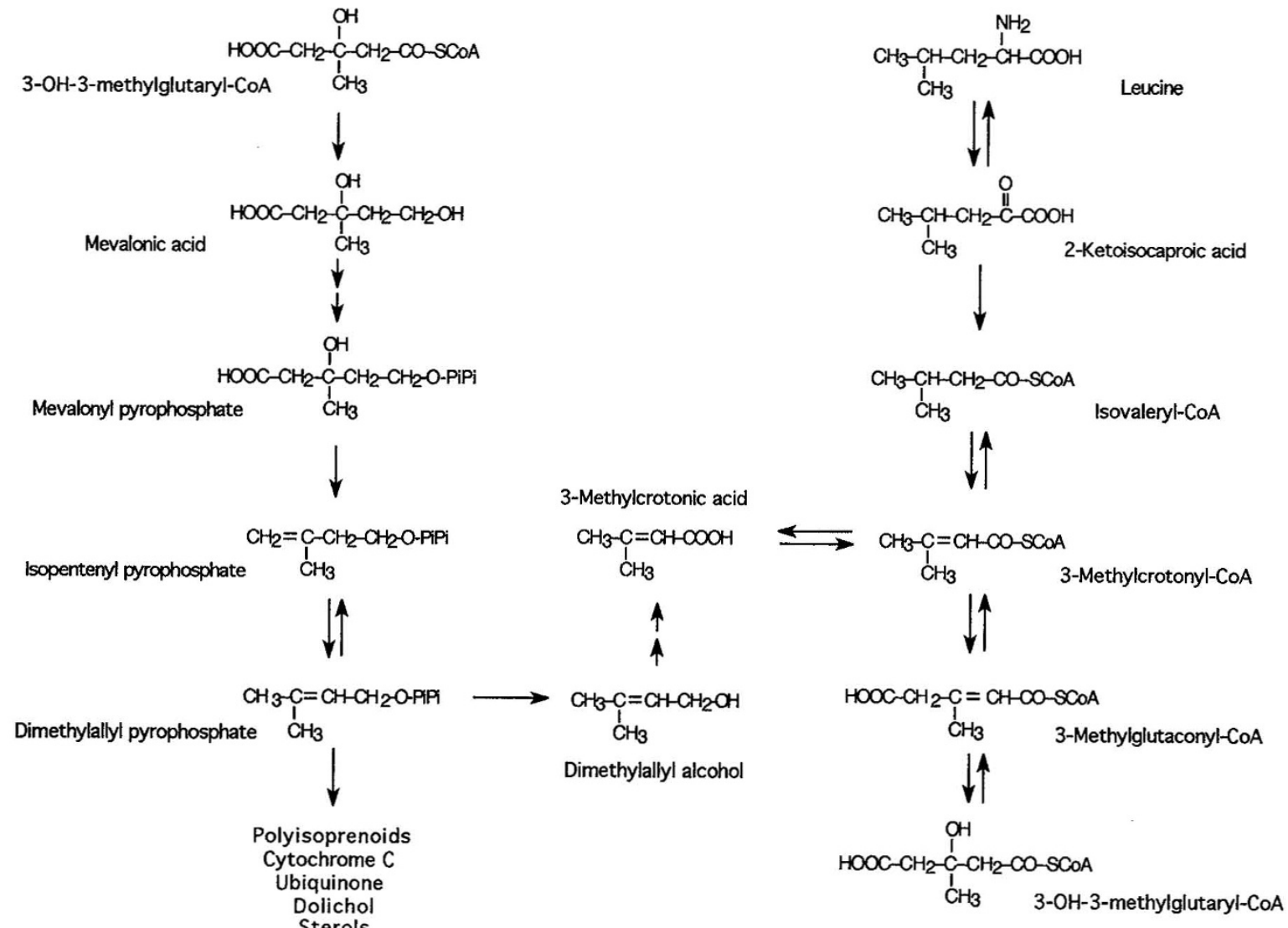

Figure 1. Pathways for isoprenoid biosynthesis and leucine catabolism showing the proposed mevalonate shunt linking the two pathways at the level of 3-methylcrotonyl-CoA. The diagram indicates two separate pools of 3-hydroxy-3-methylglutaryl-CoA, one for the microsomal/peroxisomal isoprenoid pathways (left) and one for the mitochondrial leucine catabolic pathway (right).

SLOS might lead to increased amounts of 3MGC by "overflow" of isoprenoids through the mevalonate shunt. Accordingly, we decided to measure the level of $3 \mathrm{MGC}$ in the plasma of a large number of patients with SLOS and 7-dehydrocholesterolemia of varying severity.

\section{METHODS}

Heparinized plasma samples originally submitted for biochemical diagnosis of SLOS were recovered from freezer $\left(-20^{\circ} \mathrm{C}\right)$ storage. All 42 patients with SLOS in the study had abnormally increased levels of 7DHC, which varied from 20 to 1000 times normal (16). Patients with both clinically defined extremes of severity of SLOS-classic type I (13) and severe type II (17) - as well as patients of intermediate severity were represented in the study sample. All except two teenage patients with SLOS were less than $10 \mathrm{y}$ old. Reference plasma specimens were archived samples from either sibling controls of patients with disorders other than SLOS or patients ultimately determined not to have a recognizable metabolic disease.

The levels of plasma sterols in patients with SLOS were measured by a standard ion-ratio gas chromatography/mass spectrometry technique, with minor modification, using $20-\mu \mathrm{L}$ plasma samples and epicoprostanol as the internal standard $(18,19)$. The level of $3 \mathrm{MGC}$ in plasma was measured by stable isotope-dilution gas chromatography/mass spectrometry in
400- $\mu \mathrm{L}$ samples using $3-\left[2,4,6-{ }^{13} \mathrm{C}_{3}\right]$ methylglutaconic acid as the diluting standard, as described previously (20). For seven patients with SLOS, insufficient plasma for quantification of 3MGC remained after completing the initial diagnostic sterol analysis.

\section{RESULTS}

Figure $2 A$ shows the relationship between the plasma levels of 7DHC and cholesterol for children with SLOS. The level of $7 \mathrm{DHC}$ ranged from 2 to $470 \mu \mathrm{g} / \mathrm{mL}$ (normal $\pm \mathrm{SD}=0.10 \pm$ $0.05 \mu \mathrm{g} / \mathrm{mL} ; n=52$ ). In general, children with SLOS who were on diets containing moderate to large amounts of cholesterol had normal or low normal plasma levels of cholesterol and lower levels of 7DHC than children fed proprietary formulas, most of which contain very little cholesterol. The inverse relationship between the level of $7 \mathrm{DHC}$ and that of cholesterol suggests increased endogenous synthesis of 7DHC when plasma levels of cholesterol are low. When the same plasma specimens were assayed for 3MGC, a similar inverse relationship between the plasma levels of 3MGC and cholesterol was found (Fig. 2B). As the plasma level of cholesterol decreased, the level of $3 \mathrm{MGC}$ increased, reaching abnormally high levels at cholesterol levels below $200 \mu \mathrm{g} / \mathrm{mL}$. The highest plasma levels of $3 \mathrm{MGC}$ in the SLOS patients were similar in magnitude to those of patients with Barth syndrome (1) or other idiopathic forms of $3 \mathrm{MGC}$ aciduria, whose levels of 


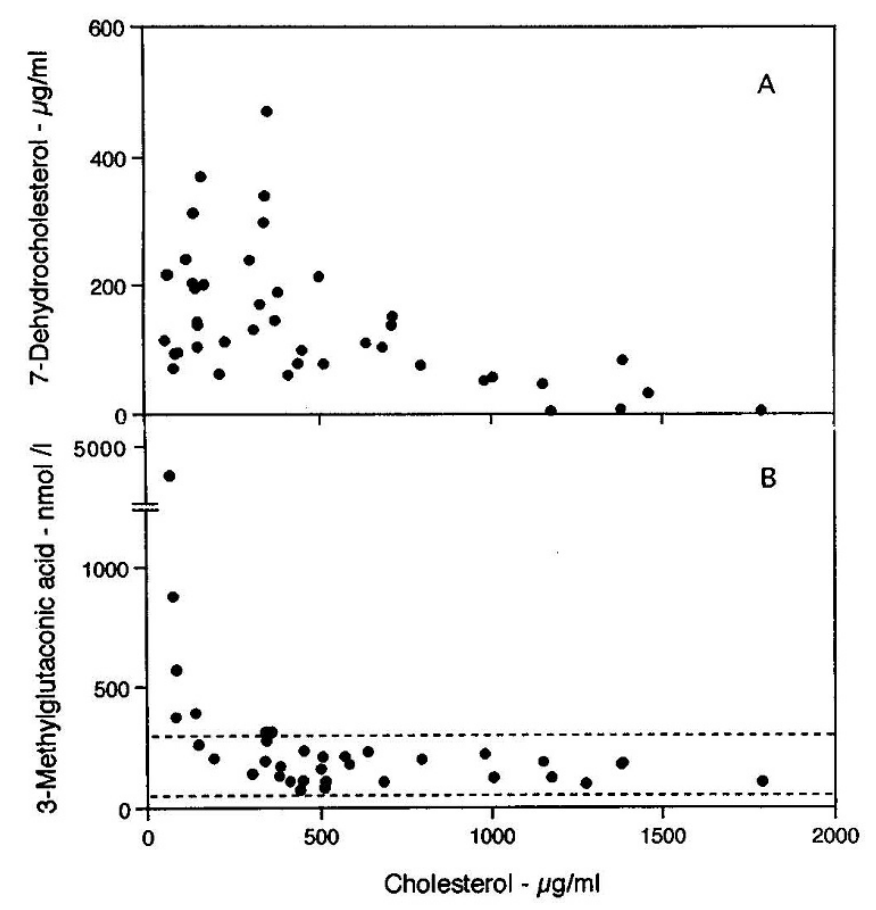

Figure 2. A, Relationship of plasma levels of cholesterol vs 7DHC in 42 patients with SLOS. $B$, Relationship of plasma levels of cholesterol vs 3MGC in 35 patients with SLOS. The horizontal broken lines demarcate normal mean \pm 2 SD for levels of $3 \mathrm{MGC}$ in plasma, age $0-10$ y (20).

3MGC in plasma typically range from 500 to $2000 \mathrm{nmol} / \mathrm{L}$ during infancy and early childhood (20). Although the younger, more severely affected SLOS patients with lower cholesterol levels had the highest levels of 3MGC, there was no correlation between age and the level of $3 \mathrm{MGC}$, either in the SLOS patients or normal controls (20). Urine specimens from several SLOS patients with high plasma levels of 3MGC were also found to contain abnormally high levels of 3MGC, but not increased levels of 3-hydroxyisovaleric acid, which are associated with forms of 3MGC-aciduria caused by abnormal leucine catabolism (3).

\section{DISCUSSION}

The results presented here are consistent with the hypothesis that some patients with idiopathic 3MGC aciduria suffer from inborn errors of sterol or polyisoprenoid biosynthesis leading to overflow of precursor metabolites through the mevalonate shunt or other linking pathway to 3MGC (Fig. 1). Theoretically, because HMG-CoA synthase can be induced by low cholesterol levels (21), the increased levels of 3MGC in patients with SLOS and hypocholesterolemia could also be caused by increased levels of HMG-CoA which is then dehydrated to 3MGC-CoA by reversal through 3-methylglutaconyl$\mathrm{CoA}$ hydratase. However, in the absence of evidence for a cytoplasmic form of 3MGC-CoA hydratase, this mechanism would require exchange between the cytoplasmic and mitochondrial pools of HMG-CoA. Although we have not found abnormal proportions of cholesterol and its neutral sterol precursors in any of our patients with idiopathic 3MGC-aciduria (Kelley R, unpublished observations), there remain numerous other possible defects of polyisoprenoid, dolichol, ubiquinone, or bile acid biosynthesis that could lead to increased levels of precursors in the pathway and, thereby, increased synthesis of 3MGC.

There is little question that patients with idiopathic 3MGCaciduria suffer from diverse genetic defects (2). Lactic acidosis or other evidence of mitochondrial dysfunction occur in some patients with idiopathic 3MGC-aciduria $(1,2)$. Similarly, several patients with well-defined primary mitochondrial disorders, such as ATP-synthase deficiency and Pearson syndrome, have been found to excrete excessive amounts of $3 \operatorname{MGC}(5,6)$, suggesting that mitochondrial dysfunction may be primary in some patients with idiopathic 3MGC aciduria. In such patients, one mechanism for increased $3 \mathrm{MGC}$-aciduria could be failure of mitochondrial uptake of peroxisomally or cytoplasmically synthesized 3MGC. On the other hand, because ubiquinone is, in part, a polyisoprenoid product, and because cholesterol and other polyisoprenoid derivatives are involved in the formation of mitochondrial membranes, mitochondrial abnormalities found in some patients with 3MGC-aciduria could also be secondary to a primary defect of sterol or isoprenoid metabolism. Clearly, the overall economy of 3MGC metabolism and its compartmentalization within cells and among tissues must be better understood before we can identify all of the causes of abnormal 3MGC metabolism. Nevertheless, our results indicate that a search for primary defects of polyisoprenoid and sterol metabolism among patients with idiopathic 3MGCaciduria may prove fruitful. Although cultured skin fibroblasts from several patients with idiopathic $3 \mathrm{MGC}$-aciduria have had grossly normal incorporation of acetate into cholesterol and other isoprenoids (22), so too have cells from patients with a profound deficiency of mevalonate kinase (23). Thus, there is a need for more comprehensive quantitative analysis of metabolites of isoprenoid and sterol biosynthesis before we can test adequately the hypothesis that genetic defects of isoprenoid and sterol biosynthesis other than SLOS are a cause of $3 \mathrm{MGC}$ aciduria.

Acknowledgment. The authors thank Catherine Peters for expert technical assistance.

\section{REFERENCES}

1. Kelley RI, Cheatham JP, Clark BJ, MD, Nigro MN, Powell B, Sherwood GW, Sladky JT, Swisher WP 1991 X-linked dilated cardiomyopathy with neutropenia, growth retardation, and 3-methylglutaconic aciduria. J Pediatr 119:738-747

2. Gibson KM, Elpeleg ON, Jakobs C, Costeff H, Kelley RI 1993 Multiple syndromes of 3-methylglutaconic aciduria. Pediatr Neurol 9:120-124

3. Narisawa K, Gibson KM, Sweetman L, Nyhan WL, Duran M, Wadman S 1986 Deficiency of 3-methylglutaconyl-coenzyme A hydratase in two siblings with 3-methylglutaconic aciduria. J Clin Invest 77:1148-1152

4. Gibson KM, Lee CF, Wappner RS 1992 3-Methylglutaconyl coenzyme A hydratase deficiency: a new case. J Inherit Metab Dis 15:363-366

5. Holme E, Greter J, Jacobson CE, Larsson NG, Lindstedt S, Nilsson KO, Oldfors A, Tulinius M 1992 Mitochondrial ATP-synthase deficiency in a child with 3-methylglutaconic aciduria. Pediatr Res 32:731-735

6. Gibson KM, Bennett MJ, Mize CE, Jakobs C, Rotig A, Munnich A, Lichter-Konecki U, Trefz FK 1992 3-Methylglutaconic aciduria associated with Pearson syndrome and respiratory chain defects. J Pediatr 121:940-942

7. Edmond J, Popjak G 1974 Transfer of carbon atoms from mevalonate to n-fatty acids. J Biol Chem 249:66-71

8. Schroepfer GJ 1981 Sterol biosynthesis. Annu Rev Biochem 50:585-621

9. Brady PS, Scofield RF, Schumann WC, Ohgaku S, Kumaran K, Margolis JM, Landau BR 1982 The tracing of the pathway of mevalonate's metabolism to other than sterols. J Biol Chem 257:10742-10746 
10. Weinstock SB, Kopito RR, Endemann G, Tomera JF, Marinier E, Murray DM, Brunengraber H 1984 The shunt pathway of mevalonate metabolism in the isolated perfused rat liver. J. Biol Chem 259:8939-8944

11. Landau BR, Brunengraber H 1985 Shunt pathway of mevalonate metabolism. Meth Enzymol 110:100-112

12. Hughes-Fulford M, Feingold KR, Searle GL, Wu J, Siperstein MJ, Schoenfeld PY Kaysen G 1986 Role of the kidneys in the metabolism of circulating mevalonate in humans. J Clin Endocrinol Metab 62:1227-1231

13. Smith DW, Lemli L, Opitz JM 1964 A newly recognized syndrome of multiple congenital abnormalities. J Pediatr 64:210-217

14. Kandutsch AA, Russell AE 1960 Preputial gland tumor sterols III: a metabolic path from lanosterol to cholesterol. J Biol Chem 235:2256-2261

15. Tint GS, Irons M, Elias E, Batta AK, Frieden R, Chen TS, Salen G 1994 Defective cholesterol biosynthesis associated with the Smith-Lemli-Opitz syndrome. N Engl J Med 330:107-113

16. Kelley RI, Moser A, Natowicz M 1994 The clinical and biochemical spectrum of 7-dehydrocholesterolemia: Smith-Lemli-Opitz syndrome and its variants. Am J Med Genet 50:335

17. Curry CJ, Carey JC, Holland JS, Chopra D, Fineman R, Golabi M, Sherman S, Pagon RA, Allanson J, Shulman S, Barr M, McGravey V, Dabiri C, Schimke N, Ives E, Hall BD 1987 Smith-Lemli-Opitz syndrome-type II: multiple congenital anomalies with male pseudohermaphroditism and frequent early lethality. Am J Med Genet 26:45-57
18. Koopman BJ, van der Molen JC, Wolthers BG, de Jager AEJ, Waterreus RJ, Gips CH 1984 Capillary gas chromatographic determination of cholestanol/cholesterol ratio in biological fluids. Its potential usefulness for the follow-up of some liver diseases and its lack of specificity in diagnosing CTX (cerebrotendinous xanthomatosis). Clin Chim Acta 137:301-315

19. Kelley RI 1995 Diagnosis of Smith-Lemli-Opitz syndrome by quantification of 7-dehydrocholesterol in plasma, amniotic fluid, and cultured skin fibroblasts using gas chromatography/mass spectrometry. Clin Chim Acta (in press)

20. Kelley RI 1993 Quantification of 3-methylglutaconic acid in urine, plasma, and amniotic fluid by gas chromatography/mass spectrometry. Clin Chim Acta 220:157164

21. Goldstein JL, Brown MS 1990 Regulation of the mevalonate pathway. Nature 343:425-430

22. Gibson KM, Sherwood WG, Hoffmann GF, Stumpf DA, Danzani I, Schutgens RBH, Barth PG, Weismann U, Bachman CB, Schrynemackers-Pitance P, Verloes A, Narisawa K, Mino M, Ohya N, Kelley RI 1991 Phenotypic heterogeneity in the syndromes of 3-methylglutaconic aciduria. J Pediatr 118:885-890

23. Gibson K, Hoffmann G, Schwall A, Broock RL, Aramaki S, Sweetman L, Nyhan WL, Brandt IK, Wappner RS, Lehnert W, Trefz FH 1990 3-Hydroxy-3-methylglutaryl coenzyme A reductase activity in cultured fibroblasts from patients with mevalonate kinase deficiency: differential response to lipid supplied by fetal bovine serum in tissue culture medium. J Lipid Res 31:515-521 